\title{
Android based Interactive Home Automation System through Internet of Things
}

\author{
Rutuja Ekatpure D ${ }^{1}$, Jadhav Suprabha $\mathbf{J}^{2}$ \\ Savitribai Phule Pune University, JSPM's JSCOE, Handewadi Road, Hadapsar, Pune, India ${ }^{1}$ \\ Assistant Professor, Electronics \& Telecommunication Engineering, Dr. Daulatrao Aher College of Engineering, \\ Karad, Maharshtra, India ${ }^{2}$
}

\begin{abstract}
Now a day's Automated systems are taking over manual system. Home Automation system using IoT is a system that uses laptops or smart phones to control the basic home functions and features using internet from anywhere around the world. It is used to save the electricity and human energy. Home automation is the automatic control and monitoring of household appliances and residential house features like TV, fans, lights, doors, gate and even the windows. Events can be programmed to be triggered under specific conditions (such as depending on the sensors data), and this can be used in reducing the total energy consumed by some appliances. On the other hand, the system can suggest smart task scheduling. In simple installations, domotics may be as straightforward as turning on the lights when a person enters the room. In advanced installations, rooms can sense not only the presence of a person inside but know who that person is and perhaps set appropriate lighting, temperature, taking into account the day of the week, the time of day, and other factors.
\end{abstract}

Keywords: Internet of Things (IoT), Raspberry Pi, Arduino, Zigbee, Automation, Image Processing.

\section{INTRODUCTION}

\section{A.BACKGROUND}

Homes of the 21st century will become more and more self-controlled and automated due to the comfort it provides, especially when employed in a private home. The "Home Automation" concept has existed for many years. The terms "Smart Home", "Intelligent Home" followed and has been used to introduce the concept of networking appliances and devices in the house.

Home automation Systems (HAS) represents a great research opportunity in creating new fields in engineering, and Computing. . A home automation system is a means that allow users to control electric appliances of varying kind. HASs includes centralized control of lighting, appliances, security locks of gates and doors and other systems, to provide improved comfort, energy efficiency and security system. HASs becoming popular nowadays and enter quickly in this emerging market. However, end users, especially the disabled and elderly due to their complexity and cost, do not always accept these systems.

Many existing, well-established home automation systems are based on wired communication. This does not pose a problem until the system is planned well in advance and installed during the physical construction of the building. But for already existing buildings the implementation cost goes very high. In contrast, Wireless systems can be of great help for automation systems. With the advancement of wireless technologies such as Zigbee, Bluetooth, Wi-Fi, cloud networks in the recent past, wireless systems are used every day and everywhere.

\section{LITERATURE REVIEW}

Design of Smart Home Control System Based on Cortex-A8 and ZigBee

In [1], the system consists of three parts which includes user intelligent control terminal, embedded home gateway and home ZigBee wireless network. It can perform functions such as safety and alarm, the indoor environment testing, household electrical appliances control and intelligent lighting and other functions. Users can access Internet web to monitor the home furnishing remotely. The users can also use cell phone with Android smart furnishing control client applications to interact remotely with home furnishing device.

Home Automation Using Internet of Things

In [2], the system uses Intel Galileo that employs the integration of cloud networking, wireless communication, to provide the user with remote control of various lights, fans, and appliances within their home and storing the data in the cloud. The system will automatically change on the basis of sensors' data. The designed system not only monitors the sensor data, like temperature, gas, light, motion sensors, but also actuates a process according to the requirement, for example switching on the light when it gets dark.

\section{An Android Based Home Automation System}

In [3], the system allows multiple users to control the appliances by an Android app or through a web site. The system has three hardware components: a local device to transfer signals to home appliances, a web server to store customer records and support services to the other 
IARJSET

components, and a mobile smart device running Android The last component is the mobile smart device running application. Distributed cloud platforms and services of Android operating system, such as smart phone or tablet, Google are used to support messaging between the on which the Android application software of the system is components.

Low cost Arduino/Android-based Energy-Efficient installed to make the mobile customers contact with and manage the in home devices via the server. The Android

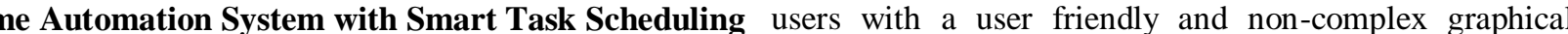
In [4],System consists of a house network (sensors and interface to easily control the automated machines at appliance actuators to respectively get information from home.

and control the house environment). House network brings together both wireless Zigbee and wired X10 technologies, Master Design: thus making it a cost-efficient hybrid system. The system uses smart scheduling.

\section{Design and Implement On Smart Home System}

In [5], the system mainly divided into the server, timing control terminal, GPRS communication terminal and cell phone control terminal. The server is mainly responsible for the management of home appliances. Timing control terminal is mainly responsible for internal home appliance's timing switch. The main task of GPRS communication terminal is remote control of home appliance. Mobile control terminal's task is home appliance's real-time controlling and getting current state of home appliance.

\section{SYSTEM ARCHITECTURE}

\section{A. PROPOSED SYSTEM}

The system mainly and simply involves the following three components:

1. Local hardware,

2. Web server, and

3. Mobile smart device.

The local hardware includes Raspberry Pi and Arduino and the local network devices to connect household appliances. Raspberry Pi acts as a master and the arduinos are act as slaves. The server connects the system with the local hardware and the mobile smart devices.

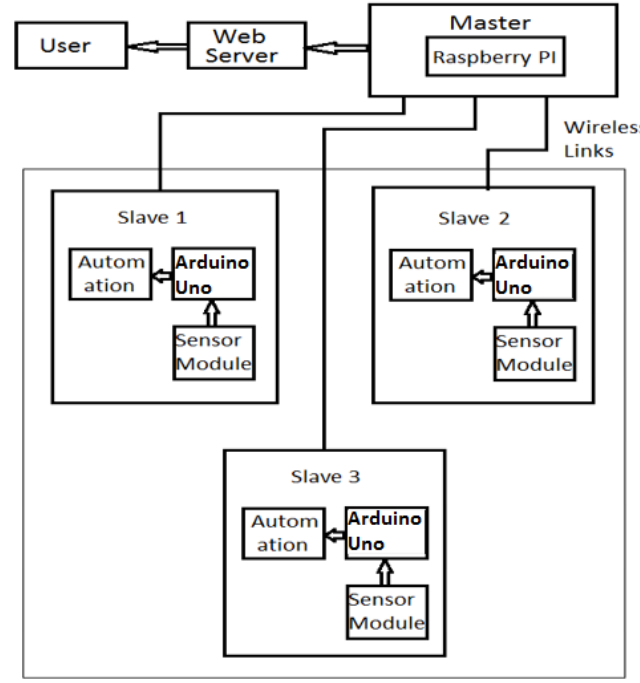

Figure 1: Detailed Design

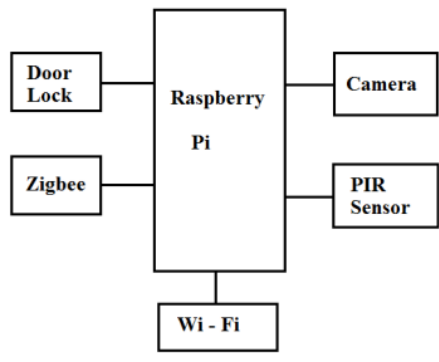

Figure 2: Design for Master

Raspberry Pi acts as a master which is placed at the entrance of the home and mainly used for

1. Transferring signal from android device to the arduino's.

2. Home security system.

Raspberry $\mathrm{Pi}$ receives command sent by the android device over the internet using the Wi-fi dongle attached to it and transfers this signal to the arduino using Zigbee.

For security system, one camera is attached to the raspberry pi. The high-level process of the security camera is that the PIR sensor will detect motion and tells the camera module snap a photo. Then raspberry uses OpenCV to decide if that photo did in fact contain a person; if the algorithm does detect a person in the photo then it stores the photo in database; else, delete the photo. Then Raspberry Pi sends alert signal to the android device and also sends the SMS with the link of the image of the person detected. Then user can send a signal to open the door to let the person come inside the home.

\section{Slave Design:}

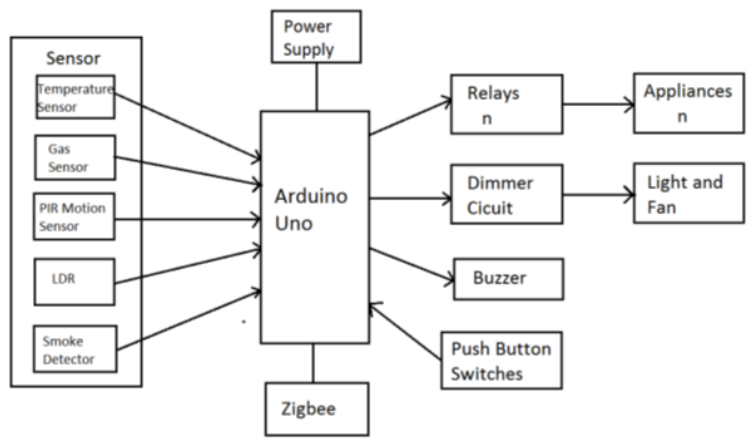

Figure 3: Design for Slave 
Arduino acts as a slave which is placed inside all the events (from sensors). In setup mode, user can add and rooms. In our project we have used two arduinos. It is mainly used for automation of the devices.

remove hardware interface modules, and can create basic Arduino receives the commands from raspberry pi using macros involving simple triggers and to customize the Zigbee. Different sensors are connected to monitor the macros to perform complex series of events. room environment which is communicated to the user through raspberry pi. All the devices are controlled using android device or through manual switches also.

Relays are used to switch ON-OFF the devices. Along with the relay, zero crossing detector is used to know the status of the device so that user can take action depending upon the current status of the device. The devices such as lights and fans are not only turned on or off, user can also control the dimming of the devices so that energy saving can be achieved.

\section{B.COMPONENT DESCRIPTION}

Raspberry Pi: The Raspberry Pi is a low cost, credit-card expect a desktop computer to do, from browsing the internet and playing high-definition video, making spreadsheets, and playing games. There are different models of Raspberry Pi from Raspberry Pi 0 to Raspberry Pi 3. In this project we are using Raspberry Pi Model 1 $\mathrm{B}+$.

Arduino: The Arduino board is a specially designed circuit board for programming and prototyping with Atmel microcontrollers. Arduino board is relatively cheap, plugs straight into a computer's USB port, and it is dead-simple to setup and use. It is an open source design. The advantage of it being open source is that it has a large community of people using and troubleshooting it.

Zigbee: ZigBee is an IEEE 802.15.4-based specification for a suite of high-level communication protocols used to create personal area networks with small, low-power digital radios. The modules operate within the ISM 2.4 $\mathrm{GHz}$ frequency band.

\section{C.SYSTEM WORKING:}

The proposed system is a distributed home automation system, consists of server, hardware interface modules. Server controls hardware interface module, and can be easily configured to handle more hardware interface module. The hardware interface module in turn controls its alarms and actuators. System can be accessed from the mobile handheld device connected to the internet.

WiFi technology is selected to be the network infrastructure that connects server and hardware interface modules. WiFi is chosen to improve system security (by using secure WiFi connection), and to increase system mobility and scalability. Even if, user intends to add new hardware interface modules out of the coverage of central access point, repeaters or managed wireless LAN will perfectly solve that problem.

Macros can be activated manually or as a reaction for certain trigger like motion sensors and surveillance cameras. User can also program macros to activate at random; this feature allows your system to turn the lights on and off at random or semi-random intervals. In running mode, if hardware interface modules report server with received events and execute their pre-programmed macros. Hardware interface modules are directly connected to sensors and actuator through direct wires connections. Hardware interface modules has the capabilities to control energy management systems like lighting, thermostats and HVAC (heating, ventilation, and cooling) systems, and security systems (door locks, cameras, motion detectors, fire alarms...).

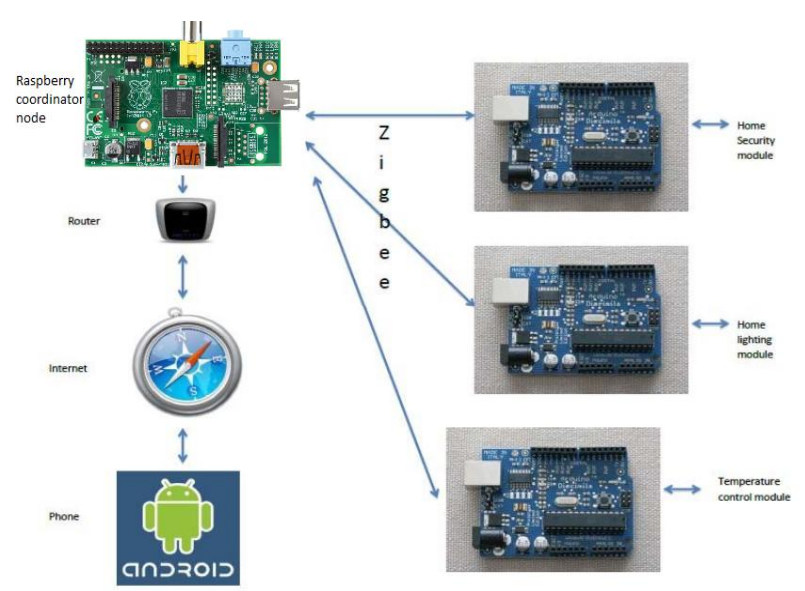

Fig 4: Pictorial Representation

\section{IMPLEMENTATION}

\section{A. SOFTWARE DESCRIPTION}

\section{Raspbian Operating System:}

Raspbian is a free operating system based on Debian optimized for the Raspberry Pi hardware. It is based on the ARM Debian 7 'Wheezy' architecture port originally designed for ARMv7 and later processors (with Jazelle RCT/ThumbEE, VFPv3, and NEON SIMD extensions), compiled for the more limited ARMv6 instruction set of the Raspberry Pi.

\section{Android Studio:}

Android Studio provides the fastest tools for building apps on every type of Android device. World-class code editing, debugging, performance tooling, a flexible build system, and an instant build/deploy system all allow you to focus on building unique and high quality apps.

The main functions of the server is to manage, control, and Android Studio is the official Integrated Development monitor distrusted system components, that enables Environment (IDE) for Android app development, based hardware interface modules to execute their assigned tasks (through actuators), and to report server with triggered on IntelliJ IDEA . On top of IntelliJ's powerful code editor and developer tools, Android Studio offers even more 
features that enhance your productivity when building building blocks for realtime interactivity. PubNub utilizes Android apps.

a Publish/Subscribe model for real-time data streaming and device signaling and supports all of the capabilities of

\section{Arduino IDE:}

The open-source Arduino Software (IDE) makes it easy to write code and upload it to the board. It runs on Windows, Mac OS X, and Linux. The environment is written in Java and based on Processing and other open-source software. This software can be used with any Arduino board.

\section{B. LANGUAGES USED}

Java: Java is a programming language first released by Sun Microsystems back in 1995. It can be found on many different types of devices from smartphones, to mainframe computers. Java doesn't compile to native processor code but rather it relies on a "virtual machine" which understands an intermediate format called Java bytecode.

Each platform that runs Java needs a virtual machine (VM) implementation. On Android the original VM is called Dalvik. The job of these virtual machines is to interpret the bytecode, which is really just a set of instructions similar to the machine code found in CPUs, and executes the program on the processor.

Python: Python, C, C++, Java, Scratch, and Ruby all come installed by default on the Raspberry Pi. The Pi in Raspberry Pi even comes from the Python programming language; the very idea of programming is reflected into the name of device. Python is a widely used highlevel, general-purpose, interpreted, dynamic programming language. Its design philosophy emphasizes code readability, and its syntax allows programmers to express concepts in fewer lines of code than would be possible in languages such as $\mathrm{C}++$ or Java. The language provides constructs intended to enable clear programs on both a small and large scale.

Embedded C: Programs written using Arduino Software (IDE) are called sketches. These sketches are written in the text editor and are saved with the file extension .ino. The editor has features for cutting/pasting and for searching/replacing text. The message area gives feedback while saving and exporting and also displays errors. The console displays text output by the Arduino Software (IDE), including complete error messages and other information.

\section{NETWORKS USED:}

Pubnub: PubNub is a secure global Data Stream Network (DSN) and easy to use API that enables to build, scale, and manage realtime functionality for applications and IoT devices. With over 60 SDKs for every platform, guaranteed $250 \mathrm{~ms}$ worldwide data transfer times, and scalability for hundreds of millions of devices, PubNub's unique infrastructure gives the ability to easily build and operate world-class realtime applications and IoT devices. PubNub enables to rapidly build and scale realtime apps by providing the cloud infrastructure, connections and key
WebSockets, Socket.IO, SignalIR, WebRTC Data Channel and other streaming protocols.

\section{Twillo:}

Twilio is a cloud based service that enables powerful communication between mobile devices, applications, services, and systems throughout the business in order to bridge the gap between conventional communication. Twilio allows programmatically making and receiving phone calls and sending and receiving text messages using its web service APIs. Twilio's services are accessed over HTTP and are billed based on usage.

\section{Imgur:}

Imgur is an image hosting and sharing site. It hosts images for free in various formats, including animated Gif files, supported by advertising, with paid-for professional accounts available. Those images are kept online for free, only deleted if the image is not accessed at least once during any six-month period. Users of the site can post comments, use tools to create new images and then share those images with both Imgur users and the wider internet.

\section{V.RESULTS}

\section{Android GUI:}

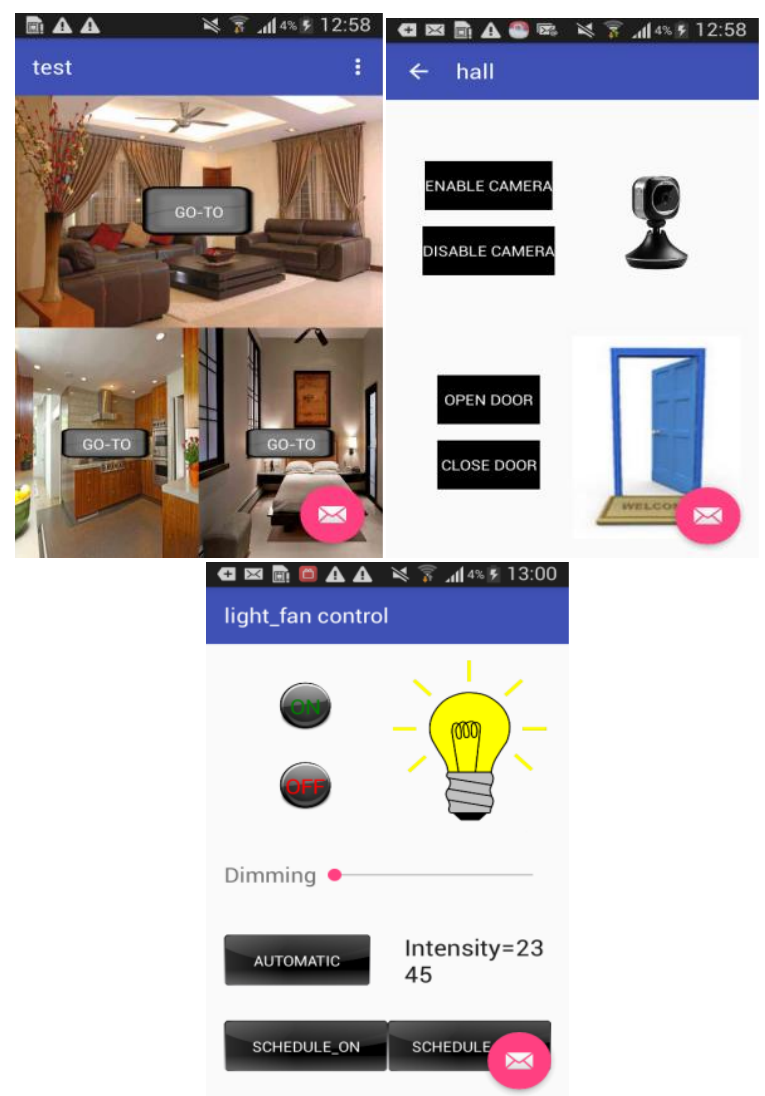

Fig 5: Pages for APP 


\section{Hardware Implementation:}

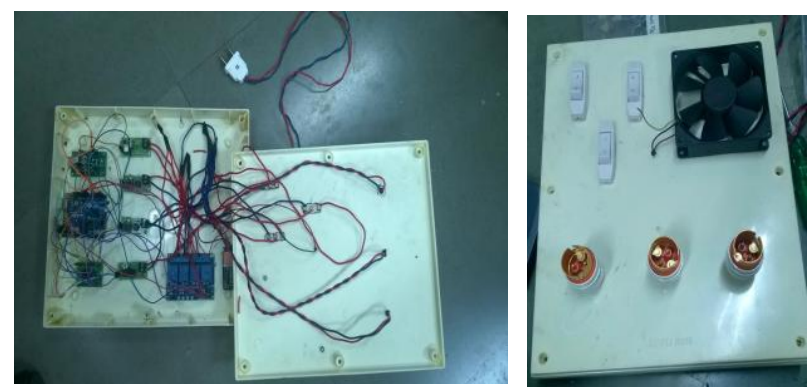

Fig 6: Device Control System

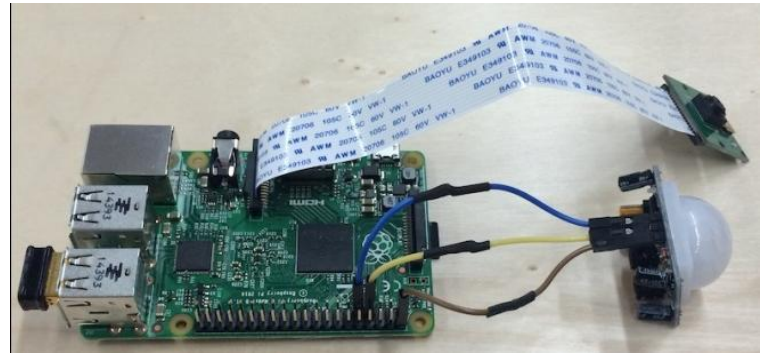

Fig 7: Security System

\section{REFERENCES}

[1] Shuyan Zhang, Pingping Xiao, Juan Zhu, Chao Wang and Xiaoguang Li, "Design of Smart Home Control System Based on Cortex-A8 and ZigBee", 978-1-4799-3279-5 /14/\$31.00 (C2014 IEEE

[2] Vinay sagar K N1, Kusuma S M2 : "Home Automation Using Internet of Things" International Research Journal of Engineering and Technology (IRJET) Volume: 02 Issue: 03 | June-2015.

[3] AlperGurek, Caner Gur, CagriGurakin, Mustafa Akdeniz, SenemKumovaMetin: "An Android Based Home Automation System” 978-1-4799-2569-8/13/\$31.00 @2013 IEEE121.

[4] Kim Baraka, Marc Ghobril, Sami Malek, Rouwaida Kanj, Ayman Kayssi, "Low cost Arduino/Android-based Energy-Efficient Home Automation System with Smart Task Scheduling”, 2013 Fifth International Conference on Computational Intelligence, Communication Systems and Networks.

[5] Shuyan Zhang, Pingping Xiao, Juan Zhu, Chao Wang and Xiaoguang Li: "Design and implement on Smart Home System" 978-1-4799-3279-5 /14/\$31.00 @2014 IEEE. 